Notre Dame Journal of Formal Logic

Volume 48, Number 1, 2007

\title{
The Borel Complexity of Isomorphism for Theories with Many Types
}

\author{
David Marker
}

\begin{abstract}
During the Notre Dame workshop on Vaught's Conjecture, Hjorth and Kechris asked which Borel equivalence relations can arise as the isomorphism relation for countable models of a first-order theory. In particular, they asked if the isomorphism relation can be essentially countable but not tame. We show this is not possible if the theory has uncountably many types.
\end{abstract}

\section{Preliminaries}

We begin by recalling the basic definitions and background material. Suppose $E_{i}$ is an equivalence relation on a standard Borel space $X_{i}$ for $i=1,2$. We say that $E_{1}$ is Borel reducible to $E_{2}$ if there is a Borel measurable $f: X_{1} \rightarrow X_{2}$ such that $x E_{1} y$ if and only if $f(x) E_{2} f(y)$ for all $x, y \in X_{1}$.

An equivalence relation is countable if every equivalence class is countable and essentially countable if it is Borel reducible to a countable equivalence relation. If $E_{1} \leq_{B} E_{2}$ and $E_{2} \leq_{B} E_{1}$, we write $E_{1} \sim_{B} E_{2}$. A Borel equivalence relation $E$ on $X$ is tame if there is a Polish space $Y$ and a Borel measurable $f: X \rightarrow Y$ such that $x E y$ if and only if $f(x)=f(y)$.

If $\mathcal{L}$ is a countable first-order language we let $X_{\mathcal{L}}$ be the Polish space of $\mathcal{L}$ structures with universe $\mathbb{N}$. For $\sigma \in \mathscr{L}_{\omega_{1}, \omega}$ let $\operatorname{Mod}(\sigma)$ be the Borel set of $\mathcal{M} \in X_{\mathscr{L}}$ with $\mathcal{M} \models \sigma$ and let $\cong_{\sigma}$ be the equivalence relation of isomorphism on $\operatorname{Mod}(\sigma)$. In general, $\cong_{\sigma}$ is $\Sigma_{1}^{1}$ but need not be Borel.

The following well-known theorem shows that $\cong_{\sigma}$ can be $\sim_{B}$ to any countable Borel equivalence relation.

Theorem 1.1 Let $\hat{E}$ be a countable Borel equivalence relation on a Polish space $X$. There is $\tau \in \mathscr{L}_{\omega_{1}, \omega}$ such that $\hat{E} \sim_{B} \cong_{\tau}$.

We give a sketch of the proof. ${ }^{1}$

Received January 20, 2006; accepted September 8, 2006; printed February 26, 2007

2000 Mathematics Subject Classification: Primary, 03C15, 03E15

Keywords: Borel equivalence relation, Scott set, $S$-saturated model

(C)2007 University of Notre Dame 
Sketch of proof Since $\hat{E}$ is a countable Borel equivalence relation, by the FeldmanMoore Theorem [2], $\hat{E}$ is the orbit equivalence relation of a Borel action of a countable discrete group $G$ on a Polish space $X$. Let $E\left(G, 2^{\omega}\right)$ be the orbit equivalence relation of the natural shift action of $G$ on $\left(2^{\omega}\right)^{G}$. There is a Borel reduction of $\hat{E}$ to $E\left(G, 2^{\omega}\right)$ (see [1], 1.2).

Let $\mathcal{L}=\{\hat{g}: g \in G\} \cup\left\{U_{n}: n \in \omega\right\}$ where each $\hat{g}$ is a unary function symbol and $U_{n}$ is a unary predicate. Let $\sigma$ be an $\mathscr{L}_{\omega_{1}, \omega^{-}}$-sentence such that $\mathcal{M}=\sigma$ if and only if $\alpha(g, x)=\hat{g}(x)$ is a faithful and transitive action of $G$ on $\mathcal{M}$.

For $\mathcal{M} \models \sigma$, we associate $f_{\mathcal{M}} \in\left(2^{\omega}\right)^{G}$ where $f_{\mathcal{M}}(g)(n)=1$ if and only if $\mathcal{M} \models U_{n}(\hat{g}(0))$. This is a Borel map from $\operatorname{Mod}(\sigma)$ to $\left(2^{\omega}\right)^{G}$ and $\mathcal{M} \cong \mathcal{N}$ if and only if $f_{\mathcal{M}} E\left(G, 2^{\omega}\right) f_{\mathcal{N}}$. Thus $\cong_{\sigma} \leq_{B} E\left(G, 2^{\omega}\right)$.

Fix $g_{0}, g_{1}, \ldots$ an enumeration of $G$ with $g_{0}=1$. For $h \in\left(2^{\omega}\right)^{G}$, construct $\mathcal{M}_{h} \models \sigma$ such that $\hat{g}_{i}(0)=i$ and $\mathcal{M}_{h} \models U_{n}(i)$ if and only if $h(i)(n)=1$. Then $f_{\mathcal{M}_{h}}=h$ and $\mathcal{M}_{h_{1}} \cong \mathcal{M}_{h_{2}}$ if and only if $h_{1} E\left(G, 2^{\omega}\right) h_{2}$. Thus $\cong_{\sigma} \sim_{B} E\left(2^{\omega}, G\right)$.

We need one lemma to complete the proof. Recall that for $E$ an equivalence relation on $X$, and $A \subseteq X$, the saturation of $A$ is $[A]_{E}=\{x: \exists y \in A x E y\}$.

Lemma 1.2 Suppose $E, F, G$ are Borel equivalence relations on Polish spaces $X, Y, Z ; E$ and $G$ are countable; and $f: X \rightarrow Y$ and $g: Y \rightarrow Z$ are Borel reductions of $E$ to $F$ and $F$ to $G$, respectively. Then $[f(X)]_{F}$ is Borel and $E \sim_{B} F \mid[f(X)]_{F}$.

Proof We make repeated use of the Lusin-Novikov Uniformization Theorem (see [4], 18.10) for Borel sets with countable sections. The map $g \circ f: X \rightarrow Z$ is countable-to-one; thus $g(f(X))$ is Borel. Since $G$ is countable, $[g(f(X))]_{G}$ is Borel. Since $[(f(X))]_{F}=g^{-1}\left([g(f(X))]_{G}\right),[f(X)]_{F}$ is Borel.

Since $E$ is countable, the set $A=\{(x, y): x \in X, f(x) F y\}$ is Borel with countable sections in $X$ and the projection of $A$ to $Y$ is $[f(X)]_{F}$. Thus there is a Borel $h:[f(X)]_{F} \rightarrow X$ such that $(h(y), y) \in A$ for all $y \in[f(X)]_{F}$. Let $y_{1}, y_{2} \in[f(X)]_{F}$. Since $f\left(h\left(y_{i}\right)\right) F y_{i}$ and $f$ is a reduction of $E$ to $F$,

$$
y_{1} F y_{2} \Leftrightarrow f\left(h\left(y_{1}\right)\right) F f\left(h\left(y_{2}\right)\right) \Leftrightarrow h\left(y_{1}\right) E h\left(y_{2}\right) .
$$

Thus $h$ is a reduction of $F \mid[f(X)]_{F}$ to $E$.

We can now finish the proof of the theorem. Since $\hat{E} \leq_{B} E\left(2^{\omega}, G\right) \sim_{B} \simeq_{\sigma}$, we can apply Lemma 1.2 to find a Borel $C \subseteq \operatorname{Mod}(\sigma)$ that is isomorphism invariant and $\hat{E} \sim_{B} \simeq_{\sigma} \mid C$. By Lopez-Escobar (see [4], 16.8) $C$ is $\operatorname{Mod}(\tau)$ for some $\mathcal{L}_{\omega_{1}, \omega^{-}}$ sentence $\tau$ and $\hat{E} \sim_{B} \simeq_{\tau}$.

Hjorth and Kechris asked if the same result is true for first-order theories. It is easy to give examples of theories $T$ with continuum many countable models where $\cong_{T}$ is tame. For example, let $T$ be the theory of an equivalence relation with infinitely many classes where each class contains an algebraically closed field. Then models are determined up to isomorphism by the set of transcendence degrees of the equivalence classes. Are there any first-order theories $T$ with $\cong_{T}$ essentially countable but not tame? We show that any such theory must have few types.

Let $\mathcal{C}$ be the Cantor space $2^{\omega}$. Fix $\langle\rangle:, \omega^{2} \rightarrow \omega$ a pairing function. For $x \in \mathcal{C}$, let $A_{x} \subseteq \mathcal{C}$ be the set $\left\{x_{1}, x_{2}, \ldots\right\}$ where $x_{i}(j)=x(\langle i, j\rangle)$. We say $x F_{2} y$ if and only if $A_{x}=A_{y}$.

The equivalence relation $F_{2}$ is not essentially countable. See [3], Exercise 2.64. 
Theorem 1.3 Let $T$ be a first-order theory in a countable language where the type space $S(T)$ is uncountable. Then $F_{2} \leq_{B} \cong_{T}$. Thus $\cong_{T}$ is not essentially countable.

This result is not surprising as the set of realized types is a natural invariant of a model.

\section{Theories with Many Types}

Suppose $T$ is a first-order theory in a countable language with $S(T)$ uncountable. We can find $\mathcal{T}$ a perfect tree of types in $S(T)$. Choose $r_{T} \in \mathcal{C}$ such that $\mathcal{L}, T, \mathcal{T} \leq_{T} r_{T}$. Using $\mathcal{T}$ we can code elements of the Cantor space as types.

Lemma 2.1 There is continuous one-to-one map $\tau: \mathcal{C} \rightarrow S(T)$ such that $\tau(x) \leq_{T} x \oplus r_{T}$ and $x \leq_{T} \tau(x) \oplus r_{T}$, where $x \oplus y$ is the join of $x$ and $y$.

\subsection{Scott sets}

Definition 2.2 We say that $S \subseteq \mathcal{C}$ is a Scott set

(i) if $x \in S$ and $y \leq_{T} x$, then $x \in S$;

(ii) if $x, y \in S$, then $x \oplus y \in S$;

(iii) if $x \in S$ codes an infinite subtree $t$ of $2^{<\omega}$, then there is $y \in S$ an infinite path through $t$.

We need a refinement of recursively saturated models.

Definition 2.3 Let $T$ be a complete first-order theory in a countable language and let $S$ be a Scott set with $T \in S$. We say that $\mathcal{M} \models T$ is $S$-saturated if

(i) for all $x \in S$ if $a_{1}, \ldots, a_{n} \in M$ and $p\left(v, a_{1}, \ldots, a_{n}\right)$ is a partial type recursive in some $x \in S$, then $p$ is realized in $\mathcal{M}$;

(ii) $\operatorname{tp}\left(a_{1}, \ldots, a_{n}\right) \in S$ for all $a_{1}, \ldots, a_{n} \in M$.

$S$-saturated models were studied in papers of Knight and Nadel ([5], [6]) and Wilmers [8]. The next result summarizes the facts that we will need.

Proposition 2.4 Let $T$ be a first-order theory in a countable language. Let $S$ be a countable Scott set with $T \in S$.

(i) There is a countable $S$-saturated model of $T$.

(ii) $S$-saturated models of $T$ are $\omega$-homogeneous.

(iii) Any two countable $S$-saturated models of $T$ are isomorphic.

The proof of (i) is a Henkin argument where one alternates trying to realize types in $S$, witnessing existential sentences and making sure that for all Henkin constants $c_{1}, \ldots, c_{n}, \operatorname{tp}\left(c_{1}, \ldots, c_{n}\right) \in S$. The uniformity of this construction (and the uniqueness of $S$-saturated models) allows us to prove the following.

Lemma 2.5 Let $\delta=\left\{x \in \mathcal{C}: A_{x}\right.$ is a Scott set $\}$. Then $s$ is Borel and there is a Borel $\mu: \& \rightarrow \operatorname{Mod}(T)$ such that $\mu(x)$ is the $A_{x}$-saturated model of $T$.

In fact, by the main result of [7], if $T \in A_{x}$, then an $A_{x}$-saturated model can be constructed recursively in $x$. 
2.2 Borel closure systems Let $\mathcal{F}=\left\{f_{1}, f_{2}, \ldots\right\}$ be a countable set of Borel functions $f_{i}: \mathcal{C}^{m_{i}} \rightarrow \mathcal{C}$. For $A \subseteq \mathcal{C}$, let $\mathrm{cl}_{\mathcal{F}}(A)$ be the closure of $A$ under the functions in $\mathcal{F}$.

Definition 2.6 We say that $I \subseteq \mathcal{C}$ is $\mathcal{F}$-independent if

$$
\operatorname{cl}_{\mathscr{F}}(A) \cap I=A
$$

for all $A \subseteq \ell$.

Lemma 2.7 For any countable set of Borel functions $\mathcal{F}$, there is a perfect $\mathcal{F}$ independent set.

Proof If $P$ is a perfect set of suitably generic Cohen reals, then $P$ is $\mathcal{F}$-independent.

Let $\mathcal{F}$ be the following collection of functions:

(i) $j(x, y)=x \oplus y$;

(ii)

$$
f_{e}(x)=\left\{\begin{array}{ll}
\varphi_{e}^{x} & \text { if } \varphi_{e}^{x} \text { is a total function in } \mathcal{C} \\
x & \text { otherwise }
\end{array} \text { for } e=0,1, \ldots\right.
$$

(iii) $t(x)=$ leftmost path in the tree coded by $x$ if $x$ codes a tree on $2^{<\omega}$ and $t(x)=x$ otherwise.

(iv) the constant function $x \mapsto r_{T}$.

If $A \subseteq \mathcal{C}$, then $\operatorname{cl}_{\mathcal{F}}(A)$ is a Scott set containing $A \cup\left\{r_{T}\right\}$. The construction of closures is uniform.

Lemma 2.8 There is a Borel $v: \mathcal{C} \rightarrow \mathcal{C}$ such that $A_{v(x)}$ is the $\mathcal{F}$-closure of $A_{x}$ for all $x \in \mathcal{C}$. In particular, $A_{v(x)}$ is a Scott set containing $A_{x} \cup\left\{r_{T}\right\}$.

2.3 Proof of Theorem 1.3 Let $P$ be a perfect $\mathcal{F}$-independent set with $\rho: \mathcal{C} \rightarrow P$ a homeomorphism. There is a Borel $\rho^{*}: \mathcal{C} \rightarrow \mathcal{C}$ such that $A_{\rho^{*}(x)}=\rho\left(A_{x}\right)$.

For $A \subseteq \mathcal{C}$ countable, let $S_{A}=\operatorname{cl}_{\mathcal{F}}(\rho(A))$ and let $\mathcal{M}_{A}$ be the unique countable $S_{A}$-saturated model of $T$.

Lemma 2.9 If $A \neq B$, then $\mathcal{M}_{A} \not \mathcal{M}_{B}$.

Proof Suppose $x \in A \backslash B$. Then $\rho(x) \in S_{A}$, but, since $P$ is $\mathcal{F}$-independent, $\rho(x) \notin S_{B}$. Since $r_{T} \in S_{A} \cap S_{B}$, it follows from Lemma 2.1, that $\tau(\rho(x)) \in S(T) \cap S_{A}$ and $\tau(\rho(x)) \notin S(T) \cap S_{B}$. The type $\tau(\rho(x))$ is realized in $\mathcal{M}_{A}$ but not $\mathcal{M}_{B}$. Thus $\mathcal{M}_{A} \neq \mathcal{M}_{B}$.

We now build our reduction of $F_{2}$ to $\cong_{T}$. For $x \in \mathcal{C}$, let $g(x)=\mu\left(\nu\left(\rho^{*}(x)\right)\right.$. Unraveling the definition,

(i) $A_{\rho^{*}(x)}=\rho\left(A_{x}\right)$;

(ii) $A_{v}\left(\rho^{*}(x)\right)=\operatorname{cl}_{\mathcal{F}}\left(\rho\left(A_{x}\right)\right)$;

(iii) $g(x)$ is a code for a $\operatorname{cl}_{\mathcal{F}}\left(\rho\left(A_{x}\right)\right)$-saturated model of $T$.

Since $S$-saturated models are unique, if $x F_{2} y$, then $g(x) \cong g(y)$. By Lemma 2.9, if $x \not 2 y$, then $g(x) \nsubseteq g(y)$. Thus $F_{2} \leq_{B} \cong_{T}$. 
Remarks Let $\operatorname{hMod}(T) \subseteq \operatorname{Mod}(T)$ be the codes for homogeneous models of $T$. Countable homogeneous models are determined by the types they realize over $\varnothing$.

Corollary 2.10 Suppose $S(T)$ is uncountable; then $F_{2} \sim_{B} \cong_{T} \mid \operatorname{hMod}(T)$.

Problem Find a first-order theory $T$ where $\cong_{T}$ is not tame and $F_{2} \not_{B} \cong_{T}$. Note that counterexamples to Vaught's conjecture have this property. Is there an $\omega$-stable theory with this property?

\section{Note}

1. I am grateful to Kechris for showing me this argument and the referee for pointing out an error in my original presentation.

\section{References}

[1] Dougherty, R., S. Jackson, and A. S. Kechris, "The structure of hyperfinite Borel equivalence relations," Transactions of the American Mathematical Society, vol. 341 (1994), pp. 193-225. Zbl 0803.28009. MR 1149121. 94

[2] Feldman, J., and C. C. Moore, "Ergodic equivalence relations, cohomology, and von Neumann algebras. I," Transactions of the American Mathematical Society, vol. 234 (1977), pp. 289-324. Zbl 0369.22009. MR 0578656. 94

[3] Hjorth, G., Classification and Orbit Equivalence Relations, vol. 75 of Mathematical Surveys and Monographs, American Mathematical Society, Providence, 2000. Zbl 0942.03056. MR 1725642. 94

[4] Kechris, A. S., Classical Descriptive Set Theory, vol. 156 of Graduate Texts in Mathematics, Springer-Verlag, New York, 1995. Zbl 0819.04002. MR 1321597. 94

[5] Knight, J., and M. Nadel, "Expansions of models and Turing degrees," The Journal of Symbolic Logic, vol. 47 (1982), pp. 587-604. Zbl 0527.03013. MR 666818. 95

[6] Knight, J., and M. Nadel, "Models of arithmetic and closed ideals," The Journal of Symbolic Logic, vol. 47 (1982), pp. 833-40. Zbl 0518.03032. MR 683158. 95

[7] Macintyre, A., and D. Marker, "Degrees of recursively saturated models," Transactions of the American Mathematical Society, vol. 282 (1984), pp. 539-54. Zbl 0557.03046. MR 732105. 95

[8] Wilmers, G., "Minimally saturated models," pp. 370-80 in Model Theory of Algebra and Arithmetic (Proceedings of the Conference, Karpacz, 1979), vol. 834 of Lecture Notes in Mathematics, Springer, Berlin, 1980. MR 606795. 95

\section{Acknowledgments}

Partially supported by NSF grant DMS-0200393. I am grateful to the logic group at Notre Dame for organizing the stimulating workshop on Vaught's Conjecture.

Department of Mathematics

University of Illinois at Chicago

Chicago IL 60607

marker@math.uic.edu 